\title{
Is the bonding of self-adhesive cement sensitive to root region and curing mode?
}

\section{Abstract}

Thaynara Faelly BOING ${ }^{1}$

Giovana Mongruel GOMES ${ }^{1}$ João Carlos GOMES ${ }^{1}$

Alessandra REIS ${ }^{1}$ Osnara Maria Mongruel GOMES ${ }^{1}$
Objectives: To evaluate the influence of two curing techniques on the degree of conversion (DC) of resin cements and on bond strength (BS) of fiber posts in different regions of root dentin. Material and Methods: Twenty single-rooted premolars were endodontically treated, and the post spaces were prepared. The roots were randomly divided into two groups $(n=10)$, according to the activation mode of the resin cement RelyX ${ }^{\mathrm{TM}}$ U200 (3M ESPE Saint Paul, MN, USA): conventional (continuous activation mode) and soft-start activation mode (Ramp). The posts (WhitePost DC/FGM) were cemented according to the manufacturer's recommendations and, after one week, the roots were crosssectioned into six discs each of 1-mm thickness, and the cervical, medium, and apical thirds of the root canals were identified. The DC was evaluated under micro-Raman spectroscopy and the BS was evaluated by the push-out test. The data were analyzed by two-way ANOVA and Tukey's test $(\alpha=0.05)$. Results: Neither the activation mode nor the root regions affected the DC of the resin cement. Higher BS was achieved in the soft-start group $(p=0.036)$; lower BS was observed in the apical third compared to the other root regions $(p<0.001)$. Irrespective of the activation mode and root region, the mixed failure mode was the most prevalent. Conclusion: The BS of fiber posts to root canals can be improved by soft-started polymerization. The DC was not affected by the curing mode.

Keywords: Resin cements. Raman spectroscopy. Dental bonding.
Corresponding address: Thaynara Faelly Boing Rua Estados Unidos, 291, Ap 93, Ponta Grossa - PR - Brazil - 84010-610 Phone: 00554299182600 - Fax: 00554232247351 e-mail: thaynaraboing@gmail.com
${ }^{1}$ Universidade Estadual de Ponta Grossa, Departamento de Odontologia, Ponta Grossa, PR, Brazil. 


\section{Introduction}

Endodontically treated teeth usually demonstrate an extensive loss of dental structure and require the use of intraradicular retainers and filling cores to hold the final restoration ${ }^{29}$. In this context, the cementation of fiber posts in endodontically treated canals results in restorations that resemble the natural dental structure because the modulus of elasticity of the adhesive materials and fiber posts are similar to that of dentin ${ }^{14}$.

Unfortunately, several factors can affect the adhesion of fiber posts to root dentin, including the histological and anatomical characteristics of the root canal, density and orientation of the dentinal tubules in the different root canal regions ${ }^{11}$, as well as accessibility to the different root canal regions ${ }^{12}$. Different areas of the same root canal also do not respond to acid etching, and thus the ability of adhesion to root dentin may be different at different depths in the same root canal ${ }^{11}$. Higher bond strength values at the cervical third are generally expected due to the ease of conditioning, and polymerization of the cements in this region'. However, this is still controversial ${ }^{3}$.

Failures in the luting process of fiber posts are still the major clinical failure ${ }^{18}$. The polymerization shrinkage of these materials may exceed their bond strength, resulting in gaps forming at the dentinresin cement interface, loss of retention and the displacement of the posts ${ }^{4}$. This may be caused by the stress that results from the polymerization shrinkage generated in the root canal by the composition of resin cements and different light curing techniques ${ }^{10,23}$.

During the pre-gel phase, the shrinkage stress is low due to the high flowability of the resin material. However, when the gel point is reached, the material's flowability is lost; then the stress generated is transferred to the remaining tooth structure, causing adhesive failures and several other adverse consequences such as tooth fracture and microcracks in the material itself 6,23 .

This situation is even worse due to the high and unfavorable cavity configuration factor (C-factor) of the root space $^{28}$. Some authors have attributed the gap formation and low bond strength to the high C-factor of the root space ${ }^{26}$. One way to control this polymerization shrinkage stress is by reducing the light intensity of the curing unit during the polymerization of the material. The use of soft-start activation provides low light intensity during the initial seconds of activation, increasing the period during which the resinous material remains in the pre-gel phase. Delaying photoactivation decreased the studied postgel shrinkage ${ }^{23}$ and polymerization shrinkage stress ${ }^{8}$.

Although this technique has shown promising results when employed in composite resin specimens ${ }^{17}$, to the extent of the author's knowledge, no study has yet evaluated this technique for fiber post cementation. Thus, this in vitro study, aimed to compare the degree of conversion and the bond strength of fiber posts in different regions of root dentin, using both conventional and soft-start polymerization techniques. The null hypothesis tested was that the degree of conversion and bond strength of the resin cement is not affected by curing mode or by root region.

\section{Material and methods}

The research project was approved by the Ethics Committee of the Dental School of the State University of Ponta Grossa, under protocol number 109.876. Twenty extracted human mandibular premolars were stored in distilled water at $4^{\circ} \mathrm{C}$ and used within 6 months from the extraction time. The inclusion criteria was that teeth were absent of restoration, caries or root cracks, absent of previous endodontic treatments, posts or crowns and absent of severe root curvatures. Further, a root length of $14 \pm 1 \mathrm{~mm}$ measured from the cement-enamel junction was required.

\section{Specimen preparation}

Teeth were transversally sectioned at the cementenamel junction using a low-speed diamond saw (Isomet 1000, Buehler, Lake Bluff, IL, USA). Endodontic access was made using a tapered fissure bur with a high-speed handpiece and water spray. The working length was established by inserting a \#10 Flexofile (Maillefer, Dentsply Ind. e Com. Ltda., Petrópolis, RJ, Brazil) into each canal until it was visible at the apical foramen. One millimeter was subtracted from this length to establish the working length. A crown-down technique was used for instrumentation with Gates Glidden drills \#2 to \#4 (Maillefer, Dentsply Ind. e Com. Ltda., Petrópolis, RJ, Brazil). Apical enlargement was performed to size 40, 0.2 taper (Maillefer, Dentsply Ind. e Com. Ltda., Petrópolis, RJ, Brazil). Irrigation was performed after every change of instrument by alternating solutions of $5 \mathrm{ml}$ of $1 \% \mathrm{NaOCl}$ and $5 \mathrm{ml}$ 
of 17\% EDTA (Biodinâmica Química e Farmacêuitca Ltda., Ibiporã, PR, Brazil) for 5 minutes. Roots were dried with paper points (Maillefer, Dentsply Ind. e Com. Ltda., Petrópolis, RJ, Brazil) and filled with a resinbased sealer (AH Plus, Dentsply DeTrey, Konstanz, Germany) and gutta-percha points using the warm vertical condensation technique. The root access was provisionally filled with conventional glass-ionomer cement (Vitro Fil LC, Nova DFL, Taquara, RJ, Brazil). The roots were stored at $37^{\circ} \mathrm{C}$ and $100 \%$ humidity for one week.

After one week, the gutta-percha was removed using the Gates Glidden burs (Maillefer, Dentsply Ind. e Com. Ltda., Petrópolis, RJ, Brazil), leaving $4 \mathrm{~mm}$ of the apical seal and the post space was prepared with a low-speed bur provided by the post manufacturer (WhitePost DC \#1, FGM, Joinvile, SC, Brazil) to a fixed depth of $10 \mathrm{~mm}$ from the cement-enamel junction. The root canals were irrigated with $10 \mathrm{~mL}$ of distilled water and dried with paper points (Maillefer, Dentsply Ind. e Com. Ltda., Petrópolis, RJ, Brazil).

\section{Experimental groups}

At this point, the teeth were randomly divided into 2 groups $(n=10)$ according to the activation mode of the resin cement in the root canal. In half of the teeth, a conventional activation mode (continuous light intensity, energy density of $40 \mathrm{~J} / \mathrm{s}$ ) was employed, while in the other half a soft-start polymerization (with initial low light intensity and an energy density of approximately $38.8 \mathrm{~J} / \mathrm{s}$ ) was employed. The light intensity of the device was measured before the beginning of the experiment using a Led Kondortech radiometer (Kondortech Equip. Odontológicos. Ltda - São Carlos, SP, Brazil). Each glass-fiber post was horizontally sectioned with a water-cooled diamond rotary cutting instrument (\#2200 diamond bur, KG Sorensen, Barueri, SP, Brazil) so that a total length of $13 \mathrm{~mm}$ remained. The fiber posts were cleaned with $70 \%$ alcohol for $5 \mathrm{~s}$. Ten millimeters of the post lengths were cemented inside the root canal, while the remaining cervical $3 \mathrm{~mm}$ served as a guide to standardize the distance of the light-curing device from the cervical root region.

All the fiber posts (WhitePost DC \#1, FGM) were cemented with a dual, self-adhesive resin cement RelyX ${ }^{\mathrm{TM}}$ U200 (3M ESPE, Saint Paul, MN, USA), which was mixed according to the manufacturer's instructions and introduced into the root canal space with a Centrix syringe (DFL, Rio de Janeiro, RJ, Brazil) before seating the fiber post. After the fiber post was seated, the excess resin cement was removed.

Then, the resin cement was immediately polymerized. In the conventional activation mode, a light intensity of $1200 \mathrm{~mW} / \mathrm{cm}^{2}$ remained constant throughout the exposure time of $40 \mathrm{~s}$. In the soft-start group, the light intensity increased linearly from 0 to $1200 \mathrm{~mW} / \mathrm{cm}^{2}$ in the first $5 \mathrm{~s}$ of exposure time and remained at $1200 \mathrm{~mW} / \mathrm{cm}^{2}$ in the next $35 \mathrm{~s}$. The light curing unit LED Raddi Plus (SDI Limited, Victoria, Australia) was employed in this experiment.

After the post luting procedures, the roots with cemented posts were covered with the conventional glass-ionomer cement, (Vitro Fil LC, Nova DFL, Taquara, RJ, Brazil), and all samples subsequently were stored in water at $37^{\circ} \mathrm{C}$ for one week.

\section{Sample preparation for Raman spectroscopy and push-out tests}

The roots were placed in separate polyvinylchloride tubes and embedded in a Duralay acrylic resin (Reliance Dental, Alsip, IL, USA). The portion of each root that contained the bonded fiber post was sectioned perpendicular to the long axis into six 1-mmthick slices. An Isomet 1000 precision cutter (Buehler, Lake Bluff, IL, USA) was used under water cooling to create two cervical, two medium, and two apical slices of each tooth. Then, all specimens were observed with a light stereomicroscope at 10X magnification in order to identify any artifacts caused by the sectioning procedure. If any defects were observed, the slices were discarded.

\section{Evaluation of the degree of conversion}

One slice of each third of all teeth was submitted to a micro-Raman spectrometer (Senterra, BrukerOptik $\mathrm{GmbH}$, Ettlingen, Germany) evaluation. After polishing and cleaning, each slice was placed under the microscope of the spectrometer. The micro-Raman spectrometer was first calibrated to zero and then to the coefficient values using a silicon sample. The following micro-Raman parameters were used: 20 $\mathrm{mW}$ neon laser with a $532 \mathrm{~nm}$ wavelength, spatial resolution of $3 \mu \mathrm{m}$, spectral resolution of $5 \mathrm{~cm}^{-1}$, accumulation time of $30 \mathrm{~s}$ with 6 co-additions, and 100x magnification (Olympus UK, London, UK) to $1-\mu \mathrm{m}$ beam diameter. In each slice, the spectra were taken from 3 random areas, and a mean of the measures was 
used to represent the degree of conversion per slice.

The Raman spectra at $1,637 \mathrm{~cm}^{-1}$ indicates unreacted $\mathrm{C}=\mathrm{C}$ double bonds of the adhesive, while at $1,608 \mathrm{~cm}^{-1}$ it represents the $\mathrm{C}-\mathrm{C}$ bonds in aromatic rings in the Bis-GMA molecules, used as internal reference peaks. The spectra of the uncured resin cement were also recorded. For this purpose the uncured resin cement was placed on a glass slide and taken for the Raman spectroscopy. The ratios of the area at 1,637 and $1,608 \mathrm{~cm}^{-1}$ for both the uncured and cured resin cement allowed for the calculation of the degree of conversion of the material according to the following equations:

(1) Rcured = Peak area $1,637 \mathrm{~cm}^{-1} /$ Peak area $1,608 \mathrm{~cm}^{-1}$

(2) Runcured $=$ Peak area $1,637 \mathrm{~cm}^{-1} /$ Peak area $1,608 \mathrm{~cm}^{-1}$

(3) Degree of conversion (\%) $=(1$ - Rcured/ Runcured) x 100

\section{Evaluation of bond strength}

All slices, including the one that was used in the micro-Raman analysis, were submitted to a push-out test. The thickness of each slice was measured with a Mitutoyo digital caliper (Kyoto, Japan) with an accuracy of $0.01 \mathrm{~mm}$. The slices were also photographed on both sides with an optical microscope (Olympus, model BX 51, Tokyo, Japan) at 40X magnification in order to calculate the cervical and apical diameters of the root canal (post + resin cement) ${ }^{14}$ for the calculation of the individual bonding areas. This measurement was taken with the UTHSCSA ImageTool 3.0 software (University of Texas Health Science Center, San Antonio, TX, USA).

Each specimen (slice) was subjected to a pushout bond strength test using a universal testing machine (AG-I, Shimadzu Autograph, Tokyo, Japan) at a crosshead speed of $0.5 \mathrm{~mm} / \mathrm{min}$. The load was applied in the apical-cervical direction until post dislodgement. Care was taken to center the push-out pin on the center of the post surface without stressing the surrounding post space walls. Different sizes of punch pins were used to match the diameter of the post at the different root thirds. Three sizes of punch pins were selected, one representative for each root canal region: cervical $(1.4 \mathrm{~mm})$, medium $(1.0 \mathrm{~mm})$ and apical $(0.6 \mathrm{~mm})$.

The maximum failure load was recorded in Newtons and converted into MPa by dividing the applied load by the bonded area (lateral area of the root canal).
The bonded area was the lateral surface of a truncated cone, and was calculated using the formula: $L S=\Pi(R$ $+r)\left[(h 2+(R-r) 2]^{1 / 2}\right.$, where $n=3.14, R=$ cervical root canal radius (cervical post + resin cement radius), $r=$ apical root canal radius (apical post + resin cement radius), and $h=$ root slice thickness ${ }^{13}$.

\section{Failure mode analysis}

After the push-out evaluation, the failure modes of all specimens were evaluated under a stereomicroscope (40X magnification), only to identify the main substrates where the failures occurred (dentin, resin cement, fiber post or mixed). Two independent and calibrated operators analyzed each fractured specimen. If any disagreement occurred between the evaluators, a consensus had to be obtained by discussion.

Then, according to the failure mode the samples were classified as either: 1 . adhesive failure between dentin and resin cement; 2 . adhesive failure between resin cement and post; 3 . cohesive failure within resin cement; 4 . cohesive failure within the post; 5 . cohesive failure within dentin; 6. mixed failure ${ }^{4,5,13,29}$.

\section{Statistical analysis}

Before running the parametric statistical analysis, we tested whether or not the assumptions of normality of the data and equality of variances were valid, using the Shapiro-Wilk and Barlett's tests at an alpha of 5\%.

The data obtained on the degree of conversion and bond strength were subjected to two-way repeated measures ANOVA and Tukey's test at a significance level of $5 \%$. The repeated factor was the root third. The fixed factors were the activation mode (continuous or soft-start) and root region (cervical, medium and apical). The data of the failure modes were compared through a chi-squared test $(a=0.05)$. All calculations were performed using the SPSS ${ }^{\circledR}$ statistical software (Statistical Package for the Social Sciences, version 21.0 Mac, SPSS Inc., Chicago, IL, USA).

\section{Results}

None of the specimens observed presented artifacts caused by the sectioning procedure, therefore all slices were tested.

\section{Degree of conversion}

Neither the main factors [activation mode $(p=0.362)$ and root region $(p=0.291)]$ nor the cross- 
Table 1- Mean and standard deviation of the degree of conversion (\%) of the resin cement using the different activation modes (continuous and soft-start) for the different root regions (cervical, medium and apical)

\begin{tabular}{lcccc}
\hline \multicolumn{1}{c}{ Activation mode } & & Root region & \\
\hline & Cervical & Medium & Apical & \\
Continuous & $72.4 \pm 6.9$ & $77.0 \pm 7.8$ & $73.6 \pm 7.0$ & $74.7 \pm 7.7$ \\
Soft-Start & $74.1 \pm 8.9$ & $78.2 \pm 5.5$ & \\
\hline
\end{tabular}

Table 2- Mean and standard deviation of bond strength (MPa) of fiber posts to root dentin using the different activation modes (continuous and soft-start) for the different root regions (cervical, medium and apical)

\begin{tabular}{lccc}
\hline \multicolumn{1}{c}{ Activation mode } & Root region & & \\
& Cervical & Medium & Apical \\
Continuous & $16.2 \pm 4.1^{\mathrm{B}}$ & $17.2 \pm 4.4^{\mathrm{B}}$ & $25.8^{ \pm \pm 7.4^{\mathrm{A}}}$ \\
Soft-Start & $20.2 \pm 4.2^{\mathrm{B}}$ & $22.1 \pm 7.4^{\mathrm{B}}$ & $26.9 \pm 6.3^{\mathrm{A}}$ \\
\hline
\end{tabular}

Means identified with the same uppercase letters are not statistically different

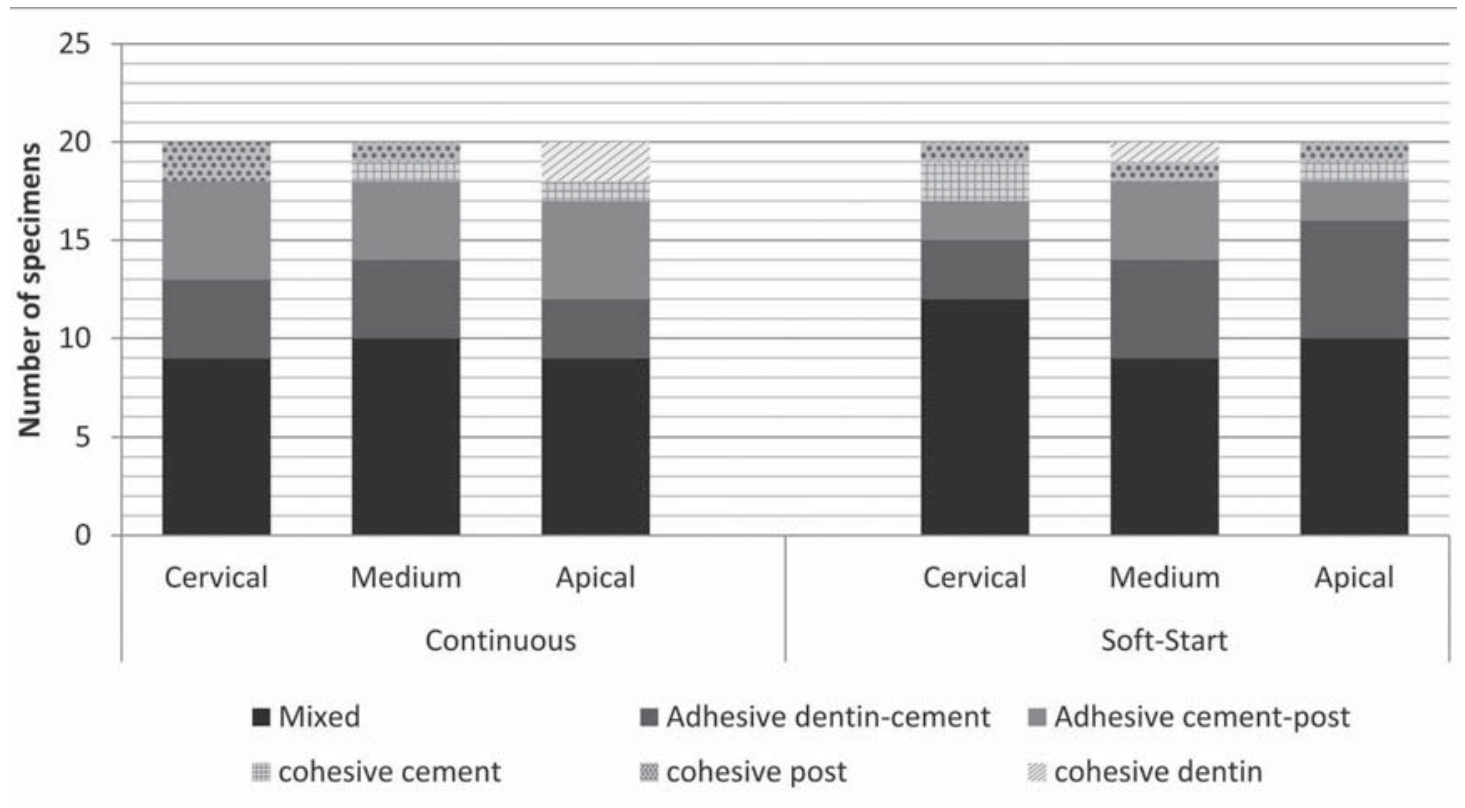

Figure 1- Absolute and Relative distribution (\%) of the failure mode, considering the activation mode and the root regions

product interaction $(p=0.949)$ influenced the degree of conversion of the resin cement. The degree of conversion of all groups was statistically similar (Table 1).

\section{Bond strength}

The average values of bond strength in $\mathrm{MPa}$ (mean and standard deviation) can be seen in Table 2 . The cross-product interaction was not significant $(p=0.634)$, but the main factors activation mode $(p=0.036)$ and root third $(p<0.001)$ were. Higher bond strength values were obtained for the soft-start activation mode and in the apical third of the root canal.

\section{Failure mode analysis}

The absolute and relative distributions (\%) of the failure modes are shown in Figure 1. Irrespective of the activation mode and root region, the mixed failure mode was the most prevalent. Representative images from the optical microscopy of each mode are illustrated in Figure 2.

\section{Discussion}

Despite recent studies that have evaluated the shrinkage stress and activation protocol associated with fiber post cementation ${ }^{6,23}$, the literature is still limited regarding the use of different curing techniques and their effects on the aforementioned properties. In the present investigation we opted to use the push out bond strength test to evaluate the strength of the bonding between the fiber posts to the root canal 

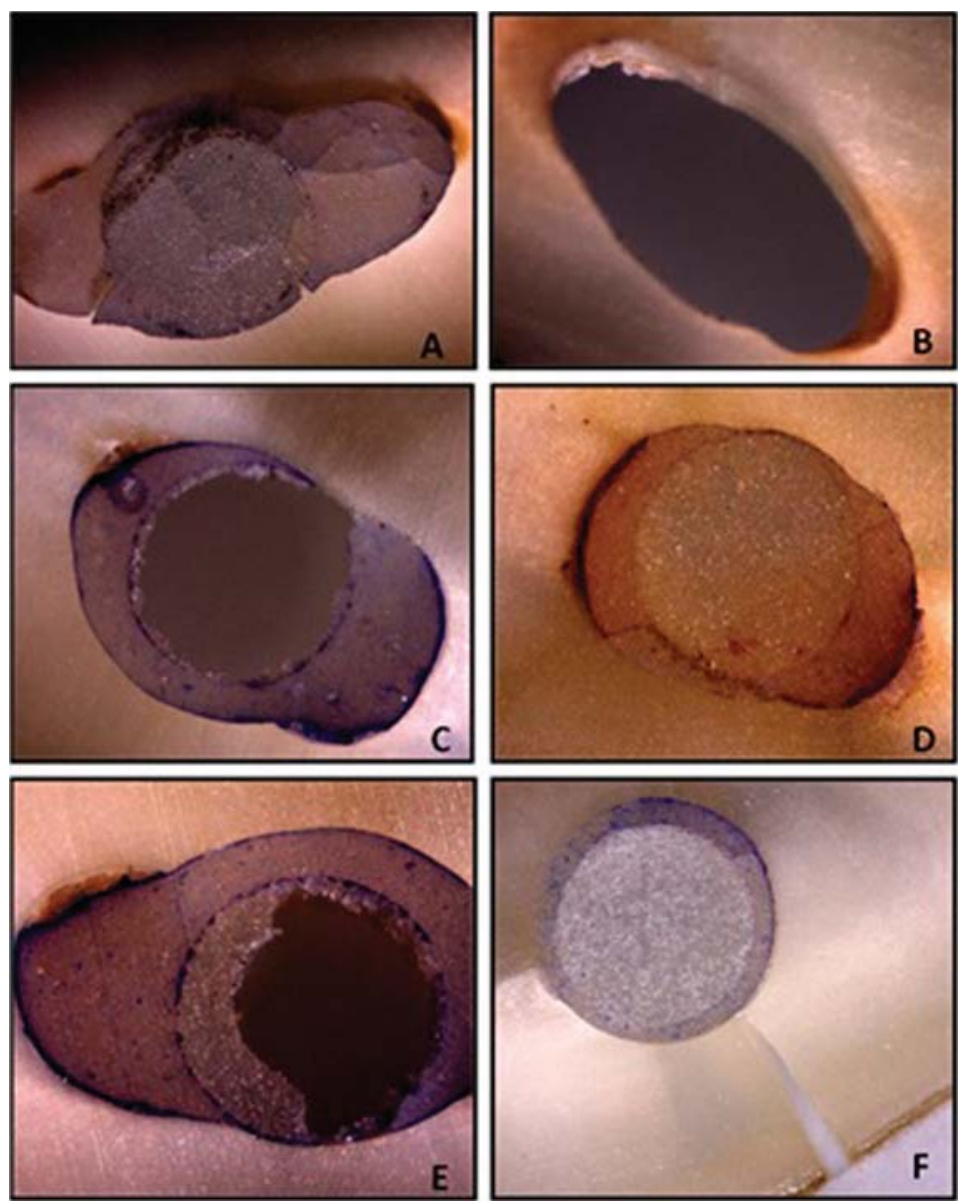

Figure 2- Optical Microscopy Representative (40X magnification) of each failure mode: A- mixed-failure; B- adhesive failure between cement-dentin; C- adhesive failure between cement-post; D- cohesive failure of cement; E- cohesive failure of post; F- cohesive failure of dentin

under varying curing techniques, as this test closely simulates the clinical condition ${ }^{15}$. According to previous studies, the push-out test provides a better estimation of the bonding strength than the conventional shear test because the fracture occurs parallel to the dentinbonding interface, which makes it a true shear test ${ }^{15}$.

Higher bond strength values were observed in the apical area of root canals irrespectively of the other factors. Although this is not in agreement with many studies published in this field?, it is in agreement with other authors ${ }^{3,22}$. The likely reason that explains such controversy is the type of resin cement employed in the different experimental designs. Studies that reported higher bond strength in the apical third ${ }^{3}$ employed self-adhesive resin cements while the others employed conventional, dual-cure luting materials ${ }^{7}$. The self-adhesive resin cement used (RelyX U200) is a new product, released as a substitute for the RelyX U100 resin cement. This resin cement has the same bonding mechanism of its predecessor, RelyX U100. Both products have micromechanical retention, but it seems that their bonding relies mainly on the chemical adhesion to hydroxyapatite ${ }^{25}$. This may be the rationale behind the product's good performance in the apical third of the root canal in the present and earlier study $^{3}$, different from what occurs with conventional resin cements.

It is known that the number of tubules greatly diminishes toward the apical region of the intraradicular dentin ${ }^{11}$. In the apical third of the root, there are fewer dentinal tubules ${ }^{11}$, the dentin is irregular, and it may be devoid of dentin tubules ${ }^{9}$. When present, these tubules are often sclerotic and filled with minerals that resemble those from peritubular dentin ${ }^{26}$. Altogether these factors increase the availability of calcium for chemical adhesion with the self-adhesive RelyX U200 in the apical region, which yields a higher bond strength at this third, as observed in this study. Conventional resin cements, on the other hand, rely mostly on micromechanical retention. Therefore, better bonding is expected to occur in areas with a high density of dentinal tubules, such as the cervical region?.

Another factor that favors self-adhesive cements, as claimed by manufacturers, is that that this type 
of resin cement was shown to be more tolerant to variations in dentin moisture, which is difficult to control in such areas of the root canal. While slight variations in the moisture degree of root dentin may jeopardize dentin bonding with conventional resin cement $^{13}$, this had not yet been demonstrated for selfadhesive resin cements. Additionally, self-adhesive resin cements have both the characteristics of resin cement and glass ionomer cement. They have a rapid polymerization reaction initiated by light irradiation, and a slow acid-base reaction between the reactive glass filler and the acid functional monomer through water ${ }^{20}$. These materials suffer from effects of water sorption and solubility, which can influence strength, biocompatibility and the dimensional and color stability of polymeric-based cements ${ }^{2}$. A slight water sorption may have an essential effect in compensating the polymerization shrinkage of the resin, thus relieving internal stresses created during shrinkage and possibly improving marginal seal by decreasing gaps ${ }^{12}$.

During the cementation of endodontic posts to root canal dentin (in the worst case scenario) the C-factor exceeds $200^{28}$. Therefore, shrinkage stress in the confinement of the intact root canal may exceed the cement dentin bond strength, causing debonding of the cement from the dentin. This is a clear limitation of bonding fiber posts to root canals. The use of soft-start polymerization has been claimed to reduce the shrinkage stress by increasing the period that the resinous material remained in a low modulus of elasticity (pre-gel phase). This is possible through the low light intensity in the first seconds of light curing, which enables an accommodation of molecules and shrinkage stress relief by reducing the speed at which the polymerization occurs ${ }^{17}$.

This probably explains the higher bond strength values observed within the groups where soft-start polymerization was employed. Although a similar degree of conversion was observed in both activation methods, in the soft-start group debonding might have occurred less often than in the continuous mode. In the continuous activation mode, the light intensity remained constant from the beginning to the end of the polymerization process, decreasing in the pre-gel phase. This in turn produced a polymeric material with reduced flow capacity and potential for stress relief. In this circumstance, the low link became the adhesive interface, which may have debonded in some areas, producing low bond strength for this group ${ }^{8,23}$.
Another technique used in this study was microRaman spectroscopy, which has been proven to be well suited for the characterization of the chemical structure and characterization of adhesive resins, collagen and minerals at a resolution of up to $1 \mathrm{~mm}$. It is also very useful in determining the degree of conversion of dental adhesives by providing a direct measurement of the percentage of converted double bonds ${ }^{16,19}$.

For light cured and dual cured resin cements, an adequate curing of the resin material by light is essential. Light intensity is higher at the cervical third ${ }^{27}$, yielding a higher degree of conversion than other regions ${ }^{9,24}$. This does not seem to be essential for self-adhesive resin cements, as the degree of conversion of Rely X U200 was neither affected by the curing technique, nor the root region. This was also observed in another published study ${ }^{29}$ that employed similar resin cement. These cited studies employed conventional, dual-cure resin cements and not selfadhesive cements, as were used in the present study.

Little has been published on the light-curing potential of conventional dual-cure cements. Earlier research suggests that auto-cure alone is not sufficient to achieve maximum cement hardening ${ }^{7}$ and this was also seen to be true for more recent resin-luting cements $^{21}$. Perhaps, the dual-cure self-adhesive resin cements are capable of reaching maximum mechanical properties under light or chemical cure modes, explaining the similar degree of conversion observed in the different root regions. Additionally, both groups employed very similar energy densities, which may also be the reason for the similar degree of conversion between the two groups. However, further studies should be conducted in this field.

Regarding failure modes, there was no statistically significant difference between the two activation modes and root regions. The most frequent failure mode was the mixed type, which agrees with the results of some authors ${ }^{1,13}$ who used self-adhesive resin cement. The present study evaluated only one type of resin cement (dual self-adhesive), which does not necessarily reflect the general behavior of these groups. Thus, future studies should investigate more resin cements in order to investigate the differences between the classifications.

The present study has some limitations, for instance, no thermal cycling or mechanical stress was applied. These factors may limit the direct application 
of the study results to clinical conditions. Another limitation is that only one resin cement was employed to investigate the research question. As resin cements differ in their chemical and mechanical properties, caution should be used when applying the results of the present study to other materials available on the market.

\section{Conclusion}

The bond strength of fiber posts to root canals can be improved by soft-started polymerization and the degree conversion was not affected by the curing mode of the resin cement.

\section{Acknowledgements}

The authors are very grateful to Prof. Dr. Fabio André dos Santos for his support with the statistical analyses. This study was partially supported by grant number 304105/2013-9 from the National Council for Scientific and Technological Development (CNPq, Brazil).

\section{References}

1- Aktemur Türker S, Uzunoğlu E, Yılmaz Z. Effects of dentin moisture on the push-out bond strength of a fiber post luted with different self adhesive resin cements. Restor Dent Endod. 2013;38:234-40.

2- Attar N, Tam LE, McComb D. Mechanical and physical properties of contemporary dental luting agents. J Prosthet Dent. 2003;89:127-34. 3- Bitter K, Meyer-Lueckel H, Priehn K, Kanjuparambil JP, Neumann $K$, Kielbassa AM. Effects of luting agent and thermocycling on bond strengths to root canal dentine. Int Endod J. 2006;39:809-18.

4- Bitter K, Noetzel J, Stamm O, Vaudt J, Meyer-Lueckel H, Neumann K, et al. Randomized clinical trial comparing the effects of post placement on failure rate of postendodontic restorations: preliminary results of a mean period of 32 months. J Endod. 2009;35:1477-82.

5- Cuadros-Sanchez J, Szesz A, Hass V, Patzlaff RT, Reis A, Loguercio AD. Effects of sonic application of adhesive systems on bonding fiber posts to root canals. J Endod. 2014;40:1201-5.

6- Daleprane $B$, Nemesio de Barros Pereira C, Oréfice RL, Bueno AC, Vaz RR, Moreira AN, et al. The effect of light-curing access and different resin cements on apical bond strength of fiber posts. Oper Dent. 2014;39:E93-100.

7- El-Badrawy WA, el-Mowafy OM. Chemical versus dual curing of resin inlay cements. J Prosthet Dent. 1995;73:515-24

8- Faria-e-Silva A, Boaro L, Braga R, Piva E, Arias V, Martins L. Effect of immediate or delayed light activation on curing kinetics and shrinkage stress of dual cure resin cements. Oper Dent. 2011;36:196-204. 9- Faria e Silva AL, Casselli DS, Ambrosano GM, Martins LR. Effect of the adhesive application mode and fiber post translucency on the push-out bond strength to dentin. J Endod. 2007;33:1078-81

10- Faria-e-Silva AL, Peixoto AC, Borges MG, Menezes MS, Moraes RR. Immediate and delayed photoactivation of self-adhesive resin cements and retention of glass-fiber posts. Braz Oral Res. 2014;28:1-6.
11- Ferrari M, Mannocci F, Vichi A, Cagidiaco MC, Mjör IA. Bonding to root canal: structural characteristics of the substrate. Am J Dent. 2000;13:255-60.

12- Ferrari M, Vichi A, Grandini S. Efficacy of different adhesive techniques on bonding to root canal walls: an SEM investigation. Dent Mater. 2001;17:422-9.

13- Gomes GM, Rezende EC, Gomes OM, Gomes JC, Loguercio AD, Reis $A$. Influence of the resin cement thickness on bond strength and gap formation of fiber posts bonded to root dentin. J Adhes Dent. 2014;16:71-8.

14- Goracci C, Ferrari M. Current perspectives on post systems: a literature review. Aust Dent J. 2011;56:77-83.

15- Goracci C, Tavares AU, Fabianelli A, Monticelli F, Raffaelli O, Cardoso PC, et al. The adhesion between fiber posts and root canal walls: comparison between microtensile and push-out bond strength measurements. Eur J Oral Sci. 2004;112(4):353-61.

16- Hass V, Dobrovolski M, Zander-Grande C, Martins GC, Gordillo $\mathrm{LA}$, Rodrigues Accorinte $\mathrm{ML}$, et al. Correlation between degree of conversion, resin-dentin bond strength and nanoleakage of simplified etch-and-rinse adhesives. Dent Mater. 2013;29:921-8.

17- Hofmann N, Denner W, Hugo B, Klaiber B. The influence of plasma arc vs. halogen standard or soft-start irradiation on polymerization shrinkage kinetics of polymer matrix composites. J Dent. 2003;31:38393.

18- Juloski J, Fadda GM, Monticelli F, Fajó-Pascual M, Goracci C, Ferrari $M$. Four-year survival of endodontically treated premolars restored with fiber posts. J Dent Res. 2014;19:52S-58S.

19- Navarra CO, Breschi L, Turco G, Diolosà M, Fontanive L, Manzoli $L$, et al. Degree of conversion of two-step etch-and-rinse adhesives: in situ micro-Raman analysis. J Dent. 2012;40:711-7.

20- Nicholson JW. Chemistry of glass-ionomer cements: a review. Biomaterials. 1998;19:485-94.

21- Pegoraro TA, Butignon LE, Filho WB, Pegoraro LF, Carvalho RM. Curing mode and aging affect monomer conversion and tensile strength of dual-cured resin-cements. Dent Mater. 2013;29:e52.

22- Pereira PC, Melo RM, Chaves C, Galhano GA, Bottino MA, Balducci I. The adhesive system and root canal region do not influence the degree conversion of dual resin cement. J Appl Oral Sci. 2010;18:477-81.

23- Pereira RD, Valdívia A, Bicalho AA, Franco SD, Tantbirojn D, Versluis $A$, et al. Effect of photoactivation timing on the mechanical properties of resin cements and bond strength of fiberglass post to root dentin. Oper Dent. 2015;40:E206-21.

24- Pulido CA, Oliveira Franco AP, Gomes GM, Bittencourt BF, Kalinowski $\mathrm{HJ}$, Gomes JC, et al. An in situ evaluation of the polymerization shrinkage, degree of conversion, and bond strength of resin cements used for luting fiber posts. J Prosthet Dent. 2016. pii: S00223913(16)00161-X. Epub ahead of print.

25- Radovic I, Monticelli F, Goracci C, Vulicevic ZR, Ferrari M. Self-adhesive resin cements: a literature review. J Adhes Dent. 2008; $10: 251-8$

26- Reginato CF, Oliveira AS, Kaizer MR, Jardim PS, Moraes RR. Polymerization efficiency through translucent and opaque fiber posts and bonding to root dentin. J Prosthodont Res. 2013;57:20-3.

27- Reis KR, Spyrides GM, Oliveira JA, Jnoub AA, Dias KR, Bonfantes $G$. Effect of cement type and water storage time on the push-out bond strength of a glass fiber post. Braz Dent J. 2011;22:359-64.

28- Tay FR, Loushine RJ, Lambrechts P, Weller RN, Pashley DH. Geometric factors affecting dentin bonding in root canals: a theoretical modeling approach. J Endod. 2005;31:584-9.

29- Zicari F, Couthino E, De Munck J, Poitevin A, Scotti R, Naert I, et al. Bonding effectiveness and sealing ability of fiber-post bonding. Dent Mater. 2008;24:967-77 